\title{
Valores y creencias asociadas al trabajo en estudiantes universitarios de Argentina y Chile
}

\section{Values and work related believes in Chilean and Argentinean college students}

\author{
Elena M. Zubieta*, Graciela Filippi \\ Universidad de Buenos Aires, Argentina \\ Ximena Báez \\ Universidad del Mar, Chile
}

(Recepción: Septiembre 2007 - Aceptación: Noviembre 2007)

\begin{abstract}
Resumen
El estudio indaga la relación entre Ética Protestante del Trabajo (EPT), Valores y Competitividad en estudiantes universitarios de Chile y Argentina, con el fin de dar cuenta de la variabilidad en valores y creencias en función de la carrera de estudio y los marcos culturales en términos de país de pertenencia. Se realizó un estudio descriptivo correlacional, de diferencias de grupo de diseño no experimental transversal sobre una muestra no probabilística intencional $(n=316)$ de estudiantes de las carreras de Psicología y Ciencias Económicas de la Universidad de Buenos Aires, Argentina y la Universidad del Mar de Antofagasta, Chile. Los resultados arrojan diferencias respecto del país y la carrera de estudio. Asimismo, se encontraron interesantes asociaciones entre Valores, Ética Protestante del Trabajo y Competitividad.

Palabras claves: Valores, creencias, trabajo.
\end{abstract}

\begin{abstract}
The study explores the relationship between Protestant Work Ethic (PWE), Values and Competitiveness in college students from Chile and Argentina. Main objective is to analyze values and beliefs variability due to university careers and cultural contexts in terms of country membership.

A descriptive correlation group differences research, with a non experimental transversal design was carried out based on a non probabilistic convenient sample composed by 316 students of Psychology and Economics Sciences from Buenos Aires University (Buenos Aires, Argentina) and Del Mar University (Antofagasta, Chile). Results show differences in the analyzed variables in terms of country and career. Also, interesting associations between Values, EPT and Competitiveness were found.
\end{abstract}

Key words: Values, beliefs, work.

\footnotetext{
* Correspondencia a: 1) Elena M. Zubieta, 2) Graciela Filippi. Facultad de Psicología, Universidad de Buenos Aires, Argentina. Av. Independencia 3065 (1225) Ciudad Autónoma de Buenos Aires. E-mail: ezubieta@psi.uba.ar - gfilippi@psi.uba.ar 3) Ximena Báez. Universidad del Mar, sede José Santos Ossa, Antofagasta, Chile. E-mail: xbaez@ujso.cl
} 


\section{Introducción}

Este artículo presenta los resultados de una investigación que explora los valores de estudiantes universitarios de Argentina y Chile. El objetivo general fue el de indagar la existencia de perfiles diferenciales de valores predominantes tanto al nivel de la carrera universitaria -como indicador de futura inserción profesional- como al nivel intercultural.

Como señalan Peiró, Prieto y Roe (1996), la perspectiva psicosocial del trabajo -en la que se inscribe este estudio- se fundamenta en la interacción social, la interpretación cultural que del trabajo se hace y en la construcción de símbolos que dan origen a creencias y valores compartidos (p.19). Además de la discusión acerca de la naturaleza antropológica o cultural del trabajo, la importancia que las personas conceden al trabajo en el sistema de valores colectivos y/o los valores a él asociados ha sido un abordaje de creciente interés en las últimas décadas.

Wilpert (1994) distingue cuatro dimensiones relevantes en el análisis del sentido cultural del trabajo: a) el significado filosófico-antropológico, b) el significado social objetivo, c) el papel económico y d) el significado psicológico individual. En lo que hace a su significado social objetivo, parecería que las sociedades actuales se han convertido en sociedades de trabajo aun con la paradoja de la caída del pleno empleo. Respecto del significado subjetivo y psicológico, éste se conforma a través de un complejo y múltiple proceso de socialización proporcionando a los individuos una identidad social (en Peiró, Prieto y Roe, 1996).

Ante la amenaza del fin de las sociedades del pleno empleo o el planteo del trabajo como un valor en peligro de extinción (Méda, 1998), cierto es que hay datos que evidencian cambios en los valores de trabajo y su significado en las sociedades actuales. Los datos de Inglehart $(1991,1998)$ muestran una tendencia general de transición de valores tradicionales de las sociedades industrializadas en las que predominan los económicos y de seguridad hacia otros post-materialistas con mayor preocupación por la calidad de vida. Es verdad que estos cambios se dan de manera diferencial según las regiones. Los estudios empíricos (Baguma y Furnham, 1993; Mendoza, 2004) muestran que en la actualidad son las sociedades menos desarrolladas y de mayor inequidad social las que enfatizan la importancia del trabajo junto a valores más materialistas y un mayor acuerdo con el trabajo duro. Las sociedades más desarrolladas parecen moverse a concepciones más postmaterialistas en donde el ocio y otras esferas vitales adquieren mayor relevancia (Inglehart, 1998). Lo que en otras épocas caracterizaba a las sociedades en desarrollo se traslada a las sociedades en las que muchos aspectos -como el trabajo- están aún sin resolver y aparecen como un bien básico escaso.

Estas creencias asociadas al trabajo y su importancia, se relacionan también con la denominada Ética Protestante del Trabajo. Para McClelland (1968) los valores e ideas que giran alrededor de aquella ética determinan prácticas de crianza cuyos valores de sustento son la independencia, la postergación de gratificaciones y el entrenamiento en habilidades de dominio del mundo externo que llevan a su vez a enfatizar las motivaciones de logro. Como señala Furnham (1990), aunque si bien la tesis original de Weber enfatiza elementos del Protestantismo, el concepto de Ética Protestante del Trabajo (EPT) se usa actualmente sin connotaciones religiosas para describir a las personas que ubican al trabajo como una esfera central de sus vidas. Parece haber un acuerdo general de que la EPT, en tanto sistema de creencias, está presente también entre sujetos no protestantes y que no es exclusivamente sobre el trabajo aunque las escalas de medición diseñadas evalúan creencias acerca del trabajo, el ocio y la moral asociada (Furnham, 1990, p. 495).

$\mathrm{Al}$ igual que lo señalado respecto de los valores materialistas y postmaterialistas y su énfasis diferencial según países y regiones, los datos de los estudios empíricos de los últimos años del siglo pasado y principios de este muestran que los sujetos de países ricos tienden a puntuar menos en la EPT que las personas de países del tercer mundo (Lynn, 1991; Baguma y Furnham, 1993). En términos de parámetros culturales a nivel país, las creencias asociadas a la EPT son menores en culturas más liberales y menos conservadoras (económica y socialmente); también, las ideas y valores de la EPT son menores en países más "científicos", con burocracias más grandes. Los países con alta distancia de poder y evitación de la incertidumbre tienden a mostrar mayor acuerdo con la EPT así 
como aquellos que tienen mayor desigualdad entre ricos y pobres (Furnham, 1990). Parecería ser entonces que una vez que los países alcanzan cierto nivel de desarrollo económico, la EPT declina, siendo ésta en parte la que aporta al progreso económico.

Por muchas razones, en las últimas dos décadas del siglo pasado los estudios estuvieron particularmente interesados en indagar en la presencia de las creencias de EPT en la gente joven. Mucho más actual y centrados en el abordaje psicosocial del trabajo, Peiró, Prieto y Roe (1996) destacan la importancia de la edad en las variaciones del significado del trabajo y mencionan los datos de un estudio que compara valores laborales de los mayores de cuarenta años con los menores de esa edad, sugiriendo una serie de cambios que deberían ser tomados en cuenta por las organizaciones. Mientras que la generación más joven piensa al trabajo como algo divertido, los mayores de cuarenta años lo ven como un deber y un medio para obtener los recursos económicos. Asimismo, los menores de 40 sostienen que las personas deberían progresar tan rápido como sus competencias se lo permitan, mientras que quienes tienen más de 40 años ponen a la experiencia como la vía necesaria de promoción. Finalmente, el estudio muestra que la generación más joven considera como "justo" el permitir que la gente sea diferente mientras que la generación más grande asocia a lo justo con tratar a la gente de manera equitativa (Jackson y Schuler, 1990 en Peiró, Prieto y Roe, 1996).

Dentro de esta línea, la elección de una carrera universitaria -como indicador de futuro ocupacional- también está íntimamente relacionada con el trabajo. Desde su mirada situacional y de desarrollo de la motivación, Schein (1996) sostiene que para entender los verdaderos valores y motivaciones de las personas, hay que examinar los tipos de experiencias de socialización que han tenido durante todas las etapas de la vida. Desde el estudio de la elección ocupacional y el desarrollo de carrera, Schein (1990) plantea la importancia que en las sociedades actuales tiene la ocupación de una persona y cómo el trabajo diario que satisface las necesidades más básicas es un aspecto central del concepto y calidad que las personas tienen de sí mismas. Indagando en las orientaciones que están detrás de la selección ocupacional, se ha logrado identificar un patrón de tipos ocupacionales que reflejaban factores que tenían que ver con los antecedentes de la persona pero también con los valores que las personas tenían en ese momento (Schein, 1972, 1978, 1996). Los estudios de este tipo se basan en el trabajo realizado por E.K. Strong (1943) y otras investigaciones realizadas durante varias décadas que muestran cómo diferentes ocupaciones reflejan diferentes patrones de interés (En Schein, 1996; p.77).

Prieto, Peiró, Bravo y Caballer (1996) destacan, desde una perspectiva psicosocial, que el análisis de los distintos fenómenos relacionados con el trabajo no puede pasar por alto el estudio y explicación del proceso mediante el cual una persona adquiere el rol de trabajador. En este proceso deben contemplarse tanto las distintas etapas del ciclo vital, las diferencias individuales de los sujetos y la dinámica de socialización en la que intervienen diversos agentes que transmiten y/o imponen normas sociales y culturales.

Ahmed y Rojas Méndez (1998) realizaron un estudio comparativo de valores en estudiantes de negocios chilenos y franceses-canadienses indicando que las instituciones educativas con fuerte orientación profesional intentan socializar a los estudiantes en lo que es la profesión, por lo que el grado en el que este proceso de socialización es alcanzado exitosamente puede ser entendido al examinar la escala de valores de los estudiantes. En este sentido, la elección de una carrera implica determinadas metas y expectativas así como percepciones, preferencias y motivaciones.

Las necesidades y preferencias asociadas a determinadas experiencias de aprendizajes se dan, además, en un determinado marco cultural. El contexto societal imprime una "cultura de enseñanzaaprendizaje" en la que se hacen salientes metas y prácticas deseables socialmente. La "programación mental de los sujetos" (Hofstede, 1980) en una determinada cultura provocará una configuración especial al nivel de los valores, y es en este sentido que es esperable observar que las prioridades de valores varíen según el medio ambiente de la especialidad de estudio y el contexto cultural. La antropología simbólica y cognitiva habla de "cultura subjetiva" como el conjunto de actitudes, normas, creencias y valores que orientan las conductas, y que son compartidas por los miembros de una nación 
o grupo (Páez y Zubieta, 2004). Las creencias, los roles, las normas y los valores son los principales componentes de la cultura subjetiva (Triandis, 1994).

Los estudios orientados a una mejor comprensión del trabajo desde la óptica de la psicología social se han centrado en el análisis del cambio en su significado y relevancia, enfatizando en las creencias y valores. La presente investigación sigue esa línea y objetivo e indaga la relación entre Ética Protestante del Trabajo y los Valores Humanos en población joven de estudiantes universitarios. Se intenta aportar datos empíricos a la discusión sobre la evolución hacia la postmaterialidad (Inglehart, 1998) y los valores puestos en juego (Schwartz, 2001), así como dar cuenta de la variabilidad en valores según la carrera de estudio en términos de experiencias de socialización e indicador de futura inserción profesional, y de los marcos culturales transmisores de creencias, delimitados aquí en términos de país de pertenencia.

\subsection{Valores y Creencias}

La investigación en valores en los últimos treinta años ha sido fuertemente influenciada por el trabajo de Milton Rockeach. Una de sus mayores contribuciones ha sido la de esclarecer la temática de la estructura de los sistemas de valores. Para Rockeach (1973), los valores no existen de manera aislada, y raramente una actitud o comportamiento singular son funciones de un solo valor, de modo que no intentaba estudiar solamente algunos valores sino que su meta era identificar todos los valores principales que existen a lo largo de las culturas humanas. Abordó la problemática asumiendo que los valores eran relativamente pocos en número y aunque nunca dio una respuesta precisa sugirió que el número de valores humanos deberían estar relacionados a necesidades básicas biológicas y sociales. Sus investigaciones demostraron la utilidad de examinar los efectos de los sistemas de valores en las actitudes y los comportamientos (Pastor Ramos, 1986). Muchos investigadores han usado las contribuciones de Rokeach para avanzar en el estudio de los valores, entre ellos, el trabajo de Shalom Schwartz es el que parece estar volviéndose el nuevo estándar para los investigadores en psicología social.

Schwartz (2001) está interesado en los efectos de los valores en actitudes y conductas, los orígenes de los valores en las experiencias únicas y compartidas de los sujetos, y en las diferencias transculturales en las prioridades de valores. Los define como metas deseables y transituacionales que varían en importancia y sirven como principios en la vida de una persona o de otra entidad social. Así, los valores: 1- sirven a los intereses de alguna entidad social; 2- pueden motivar a la acción dándole dirección e intensidad emocional; 3- funcionan como criterios para juzgar y justificar la acción, y, 4- se adquieren tanto a través de la socialización en los valores del grupo dominante como a través de la experiencia personal de aprendizaje. El aspecto fundamental del contenido que diferencia los valores es el tipo de meta motivacional que expresan; en este sentido, los valores representan, en forma de metas concientes, las respuestas que todos los individuos y sociedades deben dar a tres requisitos universales:

- las necesidades de los individuos en tanto organismos biológicos,

- $\quad$ los requisitos de la interacción social coordinada, y

- $\quad$ los requisitos para el correcto funcionamiento y supervivencia de los grupos

Schwartz (1992) encuentra diez tipos motivacionales de valores. Si bien no puede decirse que estos diez tipos de valores sean exhaustivos de todos los tipos reconocibles en las distintas culturas, se puede afirmar que es posible clasificar virtualmente todos los ítems encontrados en listas de valores específicos procedentes de distintas culturas en cada uno de estos diez tipos motivacionales de valores.

Estos valores en competición, se organizan en dos dimensiones bipolares: Apertura al Cambio con los de Conservación y Autopromoción con los de Autotrascendencia.

La Apertura al Cambio incluye a la auto-dirección -que alude a la importancia del pensamiento independiente y elección de la acción, creatividad, exploración- y la estimulación -que prioriza al entusiasmo por la novedad y los retos en la vida. 
La Conservación está conformada por valores como la Tradición, en que prima el respeto, compromiso y aceptación de las costumbres e ideas que proporciona la cultura tradicional o la religión. La Conformidad que apunta a la restricción de las acciones, inclinaciones e impulsos que pudiesen molestar o herir a otros y violar expectativas o normas sociales y, por último, la Seguridad en la que adquieren un lugar primordial la armonía y la estabilidad, ya sea de la sociedad toda, de las relaciones en general y del sí mismo.

La Autopromoción refiere a los valores de Logro, que enfatiza el éxito personal mediante la demostración de competencia según criterios sociales, y el Poder, que pone en primer lugar al estatus social que se tiene por sobre las personas y los recursos.

La Autotrascendencia se expresa en el valor de Universalismo en tanto comprensión, aprecio, tolerancia y protección del bienestar de todas las personas y la naturaleza, y Benevolencia, en el que es importante la preservación e intensificación del bienestar de las personas con las que uno está en contacto personal frecuente.

Existe un conjunto de relaciones dinámicas entre los tipos motivacionales de valores que surge del supuesto subyacente de que las acciones emprendidas para realizar cada tipo de valor tiene consecuencias psicológicas, prácticas y sociales que pueden entrar en conflicto o ser compatibles con la realización de otro tipo de valores. El análisis de los conflictos y compatibilidades que pueden ocurrir cuando las personas intentan realizar estos valores de forma simultánea, pueden ser la base de formulación de hipótesis sobre las relaciones entre prioridades de valores (Schwartz, 1992).

Las circunstancias de vida proveen oportunidades para perseguir o expresar algunos valores más fácilmente que otros $\mathrm{o}$, hacen que la persecución-expresión de diferentes valores sea más o menos costosa o reconfortante (Schwartz, 2005). Típicamente la gente adapta sus valores a sus circunstancias de vida y es por eso que podemos ver que las personas en trabajos que implican libertad de elección llegan a valorar más la autodirección a expensas de la conformidad (Kohn y Schooler, 1983; en Schwartz, 2005).

Como ya se mencionara, Ahmed y Rojas Méndez (1998) compararon en su estudio los valores de estudiantes de negocios chilenos y franceses y sus resultados revelaron diferencias entre los grupos de estudiantes respecto de sus imágenes de los sectores público y privado, de sus motivaciones de logro y al sistema de valores de Rokeach.

Filippi y Zubieta (2003) compararon estudiantes de Ciencias Económicas y Psicología de Buenos Aires y encontraron que los estudiantes de Ciencias Económicas enfatizaban valores relacionados con la Conservación combinados con metas de Logro y Poder, relacionadas con la Autopromoción, la búsqueda del éxito personal y el dominio sobre otros. Por su parte, los estudiantes de Psicología puntuaban más alto en Universalismo, un valor relacionado con la Autotrascendencia que destaca la aceptación de otros como iguales así como la preocupación por su bienestar.

\section{3. Ética Protestante del Trabajo y Competitividad}

La perspectiva según la cual los valores y creencias culturales pueden determinar como las personas interpretarán su contexto e influenciar su comportamiento social está asociada en parte a Max Weber. Este autor clásico proponía que las creencias culturales compartidas actúan a veces como factores determinantes. A su entender, la Reforma Protestante condujo hacia diferentes valores y a un "estilo de vida" que enfatizaba fuertemente el éxito mundano (Páez y González, 2000). Para Weber (1985) un conjunto de valores fueron predominantes en la época de la conformación socioeconómica del capitalismo moderno, conjunto que denominó como Ética Protestante del Trabajo. En oposición a la valorización del respeto, la resignación y la traslación de la felicidad individual a otro mundo, que promovía la religión católica, la fe protestante considera que el status y el prestigio se ganan trabajando y haciendo dinero para proveer a la familia, como una obligación moral. El deber con Dios está en la Tierra por lo que se sustituye la auto-subsistencia por la acumulación y el intercambio comercial. La motivación por adquirir y acumular forman un nuevo orden moral donde la racionalidad instrumental y de lucro legitiman nuevos valores éticos religiosos de la usura, el crédito, el comercio, el ahorro y la honestidad (Mendoza, 2004). 
Para Beit-Hallahmi (1979), la EPT es una variable personal que se ha comprobado está relacionada específicamente con percepciones de las personas sobre fenómenos económicos: "es una orientación hacia el trabajo que enfatiza la dedicación al trabajo duro, aplazamiento de recompensas inmediatas, la conservación de recursos [...] y la evitación de la haraganería y el derroche en cualquier forma" (p. 263). En palabras de Furnham (1990), "Los valores de la EPT influencian la forma en que la gente explica una amplia gama de eventos [...] por eso, puede ser considerada como un sistema cognitivo organizador a través del cual el mundo es percibido y explicado" (p. 182).

Estudios previos indican que el apoyo a la Ética Protestante del Trabajo (EPT) se asocia positivamente a la valoración de la ambición y la responsabilidad (Furnham, 1987) y al énfasis en el Logro (Feather, 1984). Asimismo, aquellas personas con puntuaciones altas en EPT muestran una mayor tendencia a culpar a las personas mismas por el desempleo (Furnham, 1982); a estar más en desacuerdo con medidas para paliar el desempleo (Heaven, 1990) y a actitudes más negativas hacia los pobres (Mac Donald, 1972). Existe además, un mayor acuerdo con la EPT en los identificados con políticas conservadoras -comparados con los liberales y los de izquierdas- (Furnham y Heaven, 1999).

Por su parte, Spence y Helmreich (1983) construyeron una escala para evaluar la Competitividad encontrando que la actitud competitiva se asociaba al individualismo instrumental y era más representativa de las culturas desarrolladas y capitalistas en comparación con las tradicionales. Se encontró una asociación positiva entre la Competitividad y la EPT indicando que representan conjuntos de creencias que están asociados. Furnham y Heaven (1999) indican que competición y valoración de bienes materiales conforman un mismo sistema de creencias tras corroborar que la actitud competitiva estaba asociada con una mayor valoración del dinero.

En un estudio realizado con estudiantes universitarios de la ciudad de Buenos Aires, Zubieta (2007) encontró que la actitud competitiva se asocia a ideas y actitudes expresadas en la Ética Protestante del Trabajo. En relación a los valores, la Ética Protestante del Trabajo se asocia al Logro y el Conformismo mientras que la Competitividad se relaciona con el Logro, el Poder y la Estimulación. La carrera de estudio fue la única variable sociodemográfica analizada que arrojó diferencias estadísticamente significativas mostrando a los estudiantes de Ciencias Económicas mucho más competitivos y apoyando de manera mucho más fuerte las creencias asociadas a la EPT.

Sobre la base de lo hasta aquí expuesto, se realizó un estudio empírico comparativo con el objetivo general de indagar en los perfiles diferenciales de valores tomando a la variable país como criterio cultural. Para esto se desarrollaron los siguientes objetivos específicos:

1) Evaluar si existen diferencias en los tipos de valores enfatizados y en creencias asociadas a la EPT y Competitividad entre las muestras de estudiantes chilenos y argentinos.

2) Analizar si se observan diferencias en las prioridades de valores y el énfasis en las creencias expresadas en la EPT y Competitividad entre las submuestras de estudiantes universitarios chilenas y argentinas, agrupados según la carrera de estudio.

\section{Método}

Se desarrolló un estudio de naturaleza descriptivo correlacional, de diseño no experimental transversal, con estudiantes universitarios como unidad de análisis.

\subsection{Participantes}

La muestra, no probabilística intencional, está compuesta por 316 sujetos. 225 estudiantes pertenecen a la Universidad de Buenos Aires, Argentina mientras que 91 estudian en la Universidad del Mar sede José Santos Ossa de la ciudad de Antofagasta, Chile. La mitad de la muestra corresponde a estudiantes de la Carrera de Psicología y la otra mitad a estudiantes de la Carrera de Ciencias Económicas. 
La muestra argentina está compuesta por un $48 \%$ de hombres y un $52 \%$ de mujeres. La media de edad es de 26 años (D.T: 7,03) y la mediana de 25 años. Un $48 \%$ de los participantes son estudiantes de Psicología y el 52\% restante de Ciencias Económicas.

La muestra chilena está compuesta por un $46,2 \%$ de individuos de sexo masculino y un $53,8 \%$ de sexo femenino. La media de edad es de 24,25 años (D.T: 3,14 ). El $73 \%$ pertenece a las carreras de Ciencias Económicas/Empresariales y el 27\% restante a la carrera de Psicología.

\subsection{Material y procedimiento}

Los participantes respondieron un cuestionario auto-administrado compuesto por: Escala de Valores (Schwartz, 2001), Escala VAL- Área Laboral (Casullo y Castro Solano, 2004), Escala de Competitividad (OCI, Spence y Helmreich, 1983) y la Escala de Ética Protestante del Trabajo (PWE-EPT Mirels y Garret, 1971). Un tercer apartado corresponde a datos sociodemográficos como: País de Nacimiento y residencia, Carrera que estudia, Universidad en la que estudia, Edad, Sexo y Estado Civil. En este estudio se han analizado sólo las variables País y Carrera de Estudio.

La Escala de Valores de Schwartz, de nivel individual, está compuesta por 40 ítemes del tipo Le parece importante disfrutar de los placeres de la vida. Le agrada "darse los gustos", en los que los participantes deben evaluar en qué medida lo descrito en cada ítem se parece mucho o nada a él o ella $(0=$ No se parece en nada a mí/ $5=$ Se parece mucho a mí).

La Escala Val (Casullo y Castro Solano, 2004) fue diseñada en base a los tipos motivacionales de Schwartz (1992), consta de 30 ítems que evalúan cada una de las metas en tres áreas: recreación y vida social, relaciones familiares y trabajo. En este trabajo sólo se aplicaron los diez ítems referidos al área trabajo, tales como Trabajar en mi casa, en forma independiente, en los que los participantes debían responder sobre la base de un continuo de respuesta que va de 1 (Nunca lo hice o pensé hacerlo) a 5 (Es lo que yo hago o me gusta hacer).

La escala de Ética Protestante del Trabajo (EPT) es un cuestionario diseñado para evaluar el grado de adhesión a la ética del trabajo en la sociedad actual. Se compone de 19 ítems del tipo Las buenas acciones a menudo pasan inadvertidas y quedan sin recompensa, con un continuo de respuesta de 1 (Totalmente en desacuerdo), 4 (Más o menos) a 7 (Totalmente de acuerdo).

La escala de Competitividad (OCI) es una subescala que forma parte de un cuestionario de actitud global que incluye a otras dimensiones como el dominio o control del mundo y la ética protestante del trabajo. Está compuesta por cinco afirmaciones positivas tales como Me gusta trabajar en situaciones de competición con otro, en los que los sujetos se posicionan respecto de lo que en ellos se afirma en un rango de respuesta que va de 1 (Fuerte Desacuerdo) a 5 (Fuerte Acuerdo). 
3. Resultados

3.1. Perfiles de valores

Tabla 1: Puntuaciones medias en Valores, EPT y Competitividad según país

\begin{tabular}{|c|c|c|c|c|}
\hline & País & Media & D.T. & $t$ \\
\hline \multirow[t]{2}{*}{ Conformismo } & Argentina & 2,90 & ,79 & \\
\hline & Chile & 2,99 &, 84 & \\
\hline \multirow[t]{2}{*}{ Tradición } & Argentina & 2,41 &, 84 & $-2,11^{*}$ \\
\hline & Chile & 2,63 &, 78 & \\
\hline \multirow[t]{2}{*}{ Benevolencia } & Argentina & 3,96 &, 62 & \\
\hline & Chile & 4,09 & 60 & \\
\hline \multirow[t]{2}{*}{ Universalismo } & Argentina & 3,93 &, 70 & \\
\hline & Chile & 3,95 & 69 & \\
\hline \multirow[t]{2}{*}{ Autodirección } & Argentina & 4,08 &, 57 & $-1,79(\&)$ \\
\hline & Chile & 4,21 &, 52 & \\
\hline \multirow[t]{2}{*}{ Estimulación } & Argentina & 3,34 & ,91 & $-2,09 *$ \\
\hline & Chile & 3,58 &, 85 & \\
\hline \multirow[t]{2}{*}{ Hedonismo } & Argentina & 4,06 &, 70 & $1,77(\&)$ \\
\hline & Chile & 3,90 &, 82 & \\
\hline \multirow[t]{2}{*}{ Logro } & Argentina & 3,26 & ,97 & \\
\hline & Chile & 3,19 &, 94 & \\
\hline \multirow[t]{2}{*}{ Poder } & Argentina & 2,45 & ,95 & \\
\hline & Chile & 2,52 & 1,06 & \\
\hline \multirow[t]{2}{*}{ Seguridad } & Argentina & 3,55 &, 73 & $2,19 *$ \\
\hline & Chile & 3,33 & ,95 & \\
\hline \multirow[t]{2}{*}{ AUTOTRASCENDENCIA } & Argentina & 3,94 &, 55 & \\
\hline & Chile & 4,02 &, 55 & \\
\hline \multirow[t]{2}{*}{ AUTOPROMOCIÓN } & Argentina & 3,25 & 62 & \\
\hline & Chile & 3,20 &, 72 & \\
\hline \multirow[t]{2}{*}{ CONSERVACIÓN } & Argentina & 2,95 & 60 & \\
\hline & Chile & 2,97 & 69 & \\
\hline \multirow[t]{2}{*}{ APERTURA AL CAMBIO } & Argentina & 3,72 & ,61 & $-2,33^{*}$ \\
\hline & Chile & 3,89 &, 58 & \\
\hline
\end{tabular}

$$
* \mathrm{p}<.05
$$

Al comparar las puntuaciones medias en los diez tipos de valores, como se puede observar en la Tabla 1, los estudiantes chilenos obtienen mayores puntuaciones que los estudiantes argentinos en Tradición, Autodirección y Estimulación mientras que los argentinos puntúan más en Seguridad y tendencialmente en Hedonismo. Asimismo, en relación a las subdimensiones subyacentes, son los estudiantes chilenos quienes muestran más Apertura al cambio en comparación con sus pares argentinos (ver también Gráfico 1). 
Gráfico 1: Puntuaciones medias en Valores de los estudiantes chilenos y argentinos (Diferencias Significativas)

$(0=$ no se parece a mí/ $5=$ se parece a mí)

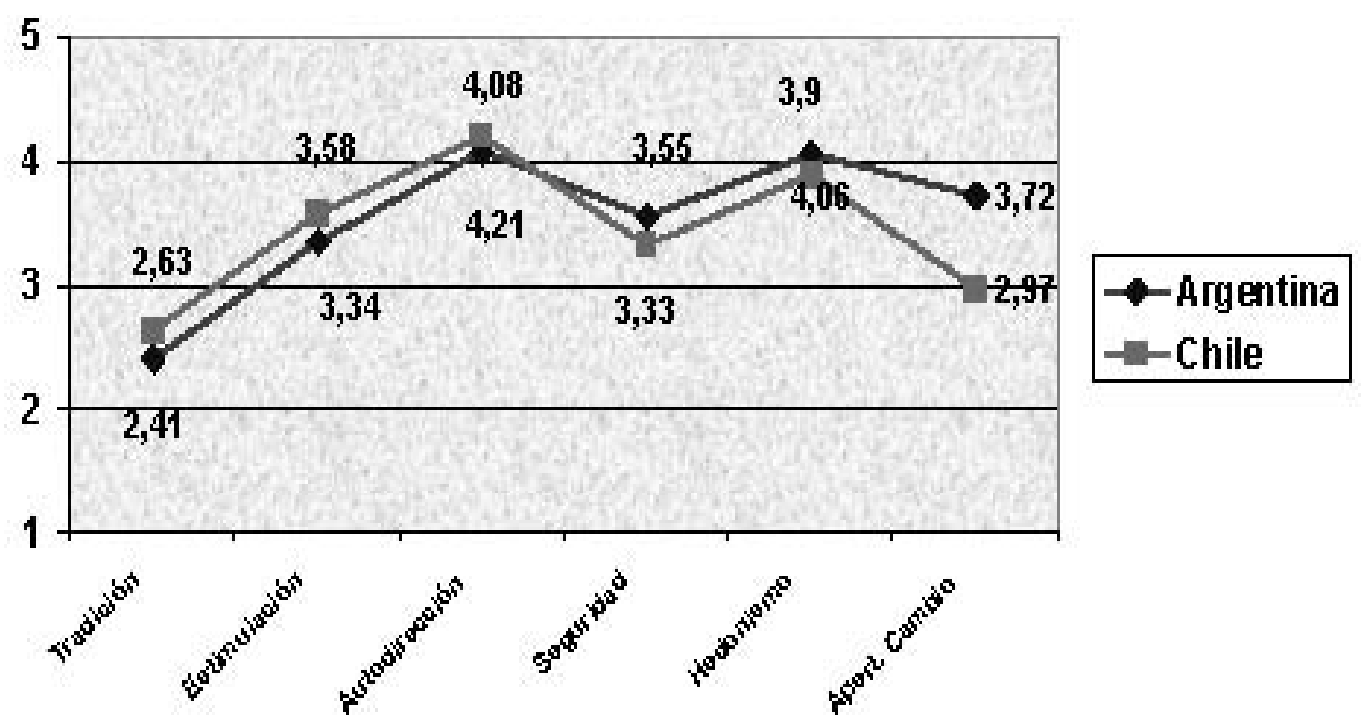

La Autodirección implica la importancia de la independencia de juicio y acción, la creatividad y la exploración, se deriva de la necesidad de control y competencia (Bandura, 1977, en Schwartz 2005) y la Estimulación enfatiza la excitación por la novedad y los desafíos en la vida. Este valor está asociado a las necesidades que subyacen a las metas de Autodirección, por eso integran la subdimensión de Apertura al Cambio y es donde los estudiantes chilenos sobresalen por sobre los argentinos. Esta importancia de la autonomía y la independencia aparece compitiendo con la Tradición en los estudiantes de Chile, que a su vez también priorizan aspectos asociados al respeto, compromiso y aceptación de las ideas o costumbres que la cultura provee. Estos valores en competencia pueden ser el reflejo de cierto movimiento hacia un mayor individualismo cultural que registra Chile a fines del siglo pasado (Fernández, 1997, en Zubieta 1998). Al igual que Japón, la prosperidad económica durante las décadas pasadas ha ido erosionando cierto colectivismo cultural hacia un mayor énfasis en el individualismo que se reflejaría en un mayor hedonismo, materialismo, pérdida de compromiso y prioridad de las necesidades individuales por sobre las comunitarias (Kelly, 1991, en Baguma y Furnham, 1993).

En lo que hace a los estudiantes argentinos, dan importancia al valor de Seguridad priorizando la armonía y estabilidad de la sociedad, de las relaciones y de uno mismo. Los valores más conservadores en los estudiantes argentinos están seguramente asociados a los efectos de la crisis económica, política y social ocurrida en Argentina en el año 2001; es sabido que la mayor incertidumbre social y el bajo nivel de desarrollo económico relativo lleva al individuo a adoptar posiciones más materialistas y conservadoras (Carballo, 2005).

Así como se observó en Argentina, entre los años 1984 y 1995, una reducción de las posiciones materialistas -predominio de valores económicos y de seguridad- y el aumento de una tendencia postmaterialista -mayor preocupación por la calidad de vida-, la tendencia se detuvo entre los años 1995 y 1999, y aún en el año 2004 eran bajas las proporciones de sujetos que daban prioridad a la libertad de expresión y la participación ciudadana, encontrándose los porcentajes más altos en la preocupación por el alza de precios y la necesidad de mantener el orden (Carballo, 2005).

Al comparar entre las submuestras de cada país de estudiantes de psicología (gráfico 2), se observa que los estudiantes chilenos de esta carrera enfatizan más los valores de Tradición (2,58 y 2,28, $\mathrm{t}(169):-2.22, \mathrm{p}<.05)$ mientras que quienes estudian psicología en Argentina enfatizan más el valor de 
Hedonismo (4,05 y 3,79, $\mathrm{t}(169): 2.16, \mathrm{p}<.05)$. El grupo de estudiantes chileno da mayor importancia al mantenimiento de las ideas y costumbres mientras que sus pares argentinos priorizan aquello relacionado con placeres y gratificaciones para uno mismo. Aquí aparecen en competencia entre estos grupos una mayor Apertura al cambio y mayor centralidad del individuo en términos de autonomía versus un cierto elemento de conservación que implica la renuncia de la autonomía por mantener aspectos fundamentales para la supervivencia del grupo de pertenencia. Se revierte en el grupo de estudiantes de psicología la tendencia descrita al comparar las muestras por países sin incluir la variable carrera de estudio.

\section{Gráfico 2: Puntuaciones medias en Tradición y Hedonismo}

de los estudiantes de Psicología de Chile y Argentina

$$
(0=\text { no se parece a mí/ } 5=\text { se parece a mí) }
$$

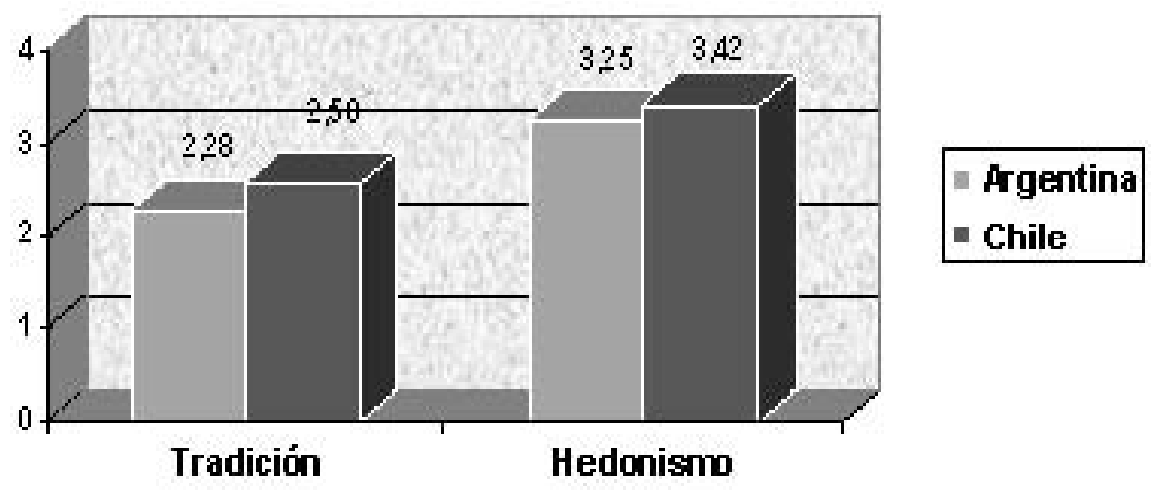

Respecto de las submuestras de los estudiantes de Ciencias Económicas y Empresariales de ambos países (gráfico 3), se observa que los estudiantes chilenos de estas carreras enfatizan más que sus pares argentinos los valores de Estimulación (3,42 y 3,93; t(138): -2,61, p<.05) y Poder (2,62 y 3,09; $\mathrm{t}(137)$ : $-2.25, \mathrm{p}<.05)$. También es mayor en el grupo chileno la puntuación en Apertura al Cambio $(3,74$ y 4,$10 ; \mathrm{t}(135):-2,78 ; \mathrm{p}<.01)$.

Aquí vemos que los estudiantes chilenos priorizan una mayor autonomía de pensamiento y acción junto con los desafíos y retos más la necesidad de poder que resalta el prestigio y el estatus social e implica la dominancia sobre personas y recursos. Hay mayor énfasis de la individualidad, de la autopromoción, en los estudiantes chilenos en comparación con los argentinos, en lo que refiere a la carrera de ciencias económicas y empresariales. 
Gráfico 3: Puntuaciones medias en Estimulación, Poder y Apertura al cambio de los estudiantes de Ciencias Económicas de Chile y Argentina

$(0=$ no se parece a mí/ $5=$ se parece a mí)

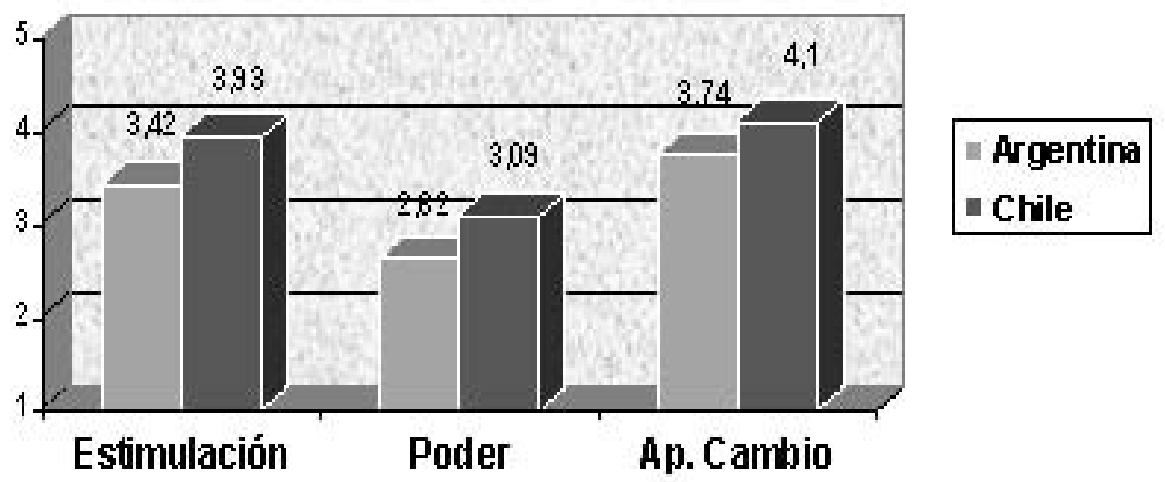

$\mathrm{Al}$ analizar las puntuaciones medias de los estudiantes de ambos países en los ítems de la Escala VAL, dimensión área laboral, se encontraron diferencias significativas en tres ítems, éstos se muestran en el gráfico 4. Como puede observarse, los estudiantes chilenos acuerdan más que los argentinos en: "trabajar más en mi casa, en forma independiente", $\mathrm{t}(311):-3,43 ; \mathrm{p}<.001)$; "ocuparme de la religión, las tradiciones y las costumbres regionales del país", (t(311): -1.92; p<.05). Por su parte, los estudiantes argentinos puntúan más en "hacer un trabajo que deje conforme a mi jefe y me asegure la continuidad laboral", (t(311): 1.98; $\mathrm{p}<.05)$.

Se ratifica aquí el mismo cuadro encontrado en las metas motivacionales ya que el ítem "Trabajar en mi casa, en forma independiente" apuntaba a la Autodirección ( $r=0,12 ; \mathrm{p}<.05)$, mientras que el ítem "Ocuparme de la religión, las tradiciones y las costumbres regionales del país" alude a la Tradición ( $\mathrm{r}=0,37 ; \mathrm{p}<.01)$. Por su parte, el ítem "Hacer un trabajo que deje conforme a mi Jefe y me asegure la continuidad laboral" se correspondía con el valor de Conformismo ( $r=0,20 ; p<.05)$ que integra la subdimensión de Conservación junto al valor o meta de Seguridad (Schwartz, 2001).

Gráfico 4: Puntuaciones medias de estudiantes de Chile y Argentina en

\section{Valores en Área del Trabajo ${ }^{1}$}

(1= Nunca lo hice o pensé hacerlo/ 5= Es lo que hago o me gusta hacer $)$

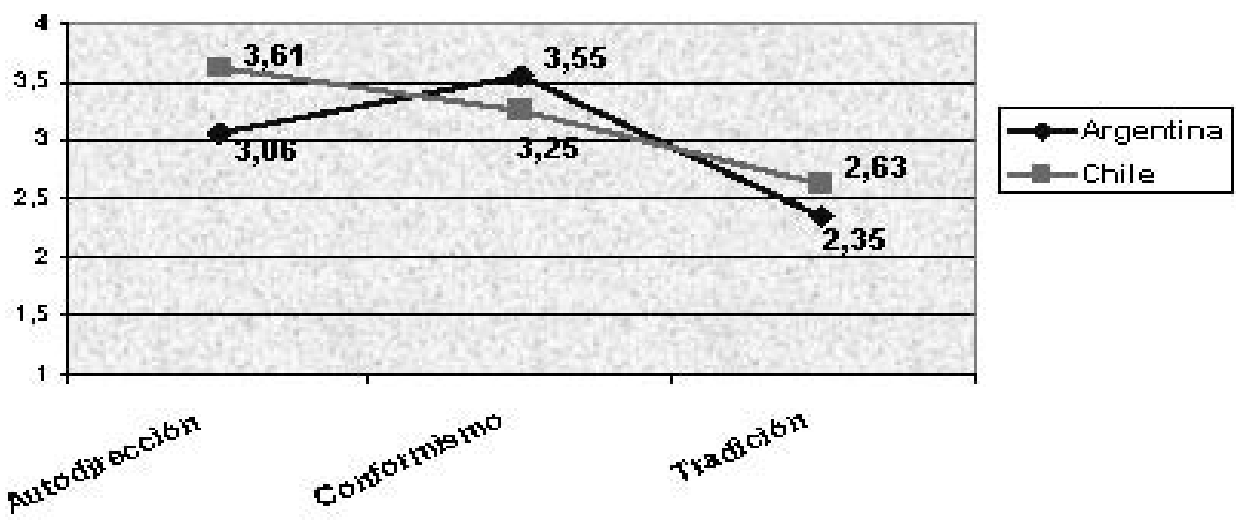

${ }^{1}$ Val 1- Trabajar en mi casa, en forma independiente

Val 6- Hacer un trabajo que deje conforme a mi Jefe y me asegure la continuidad laboral

Val 8- Ocuparme de la religión, las tradiciones y las costumbres regionales del país 
No se encontraron diferencias entre las puntuaciones de los estudiantes de psicología de ambos países aunque sí entre quienes estudian ciencias económicas. Los estudiantes chilenos de ciencias económicas puntúan más en "trabajar en mi casa, de forma independiente" -asociado a la Autodirección- que los estudiantes argentinos de esas carreras (3,96 y 2,97), respectivamente ( $\mathrm{t}(139):-3.67 \mathrm{p}<.01)$. La submuestra de estudiantes de ciencias económicas y empresariales, al igual que en un estudio previo (Zubieta, 2007) respecto al género, aparece con mayor heterogeneidad. Al incorporar la variable país, el valor que se pone en juego en cuanto a mayor prioridad es el asociado con la Autodirección y es más fuerte en los estudiantes chilenos. Este valor, asociado más a la carrera de ciencias económicas y empresariales se deriva de las necesidades de control y competencia y de los requisitos de interacción de autonomía e independencia (Schwartz, 2005).

\subsection{EPT y Competitividad}

Tabla 2 a y b: Puntuaciones Medias en Ética Protestante del Trabajo por país

$$
\text { y por submuestras de Carreras }
$$

a) $(1=$ Totalmente en Desacuerdo/ $7=$ Totalmente de Acuerdo $)$

\begin{tabular}{|c|c|c|c|}
\hline País & Media & D.T & $t$ \\
\hline Argentina & 74,98 & 14.79 & -2.78 \\
Chile & 86,91 & 15.01 & $\mathrm{p}<.01$ \\
\hline
\end{tabular}

b)

\begin{tabular}{|c|c|c|c|c|}
\hline Carrera de Estudio & País & Media & D.T & $t$ \\
\hline Psicología & Argentina & 71.48 & 12.06 & $\begin{array}{r}-2.60 \\
\mathrm{p}<.01\end{array}$ \\
\hline Ciencias Económicas & Chile & 77.12 & 14.18 & \\
\hline & Argentina & 77.54 & 16.45 & $\begin{array}{r}-2.64 \\
\mathrm{p}<.01\end{array}$ \\
\hline
\end{tabular}

En lo que hace a la escala de Ética Protestante del Trabajo, como puede observarse en las puntuaciones de la tabla 2 a y b, hay diferencias significativas tanto entre las muestras de países como entre las submuestras conformadas a partir de la carrera de estudio. Las puntuaciones hasta 75 indican una tendencia a creer poco o compartir en menor medida las ideas y valores asociados a la EPT. Tanto la muestra total como la submuestra de estudiantes de Psicología de Argentina están por debajo de esa puntuación, indicando que estos sujetos acuerdan menos con aquella concepción -sobre todo los futuros psicólogos-. Sólo las puntuaciones de los estudiantes de ciencias económicas de Argentina están por encima de 75 y son por tanto, quienes exhiben mayor tendencia a tener creencias compatibles con la EPT. Sin embargo, son las puntuaciones con un valor de 86 o más las que indican un fuerte acuerdo con la EPT. En lo que respecta a Chile, tanto la muestra total como la de los estudiantes de ciencias económicas y empresariales alcanzan ese valor. Los estudiantes de psicología quedan en un nivel intermedio al igual que sus pares de ciencias económicas en Argentina (ver gráfico 5).

Gráfico 5: Puntuaciones Medias en Ética Protestante del Trabajo 
por Países y Submuestras de Carreras

( $1=$ Totalmente en Desacuerdo/ 7= Totalmente de Acuerdo)

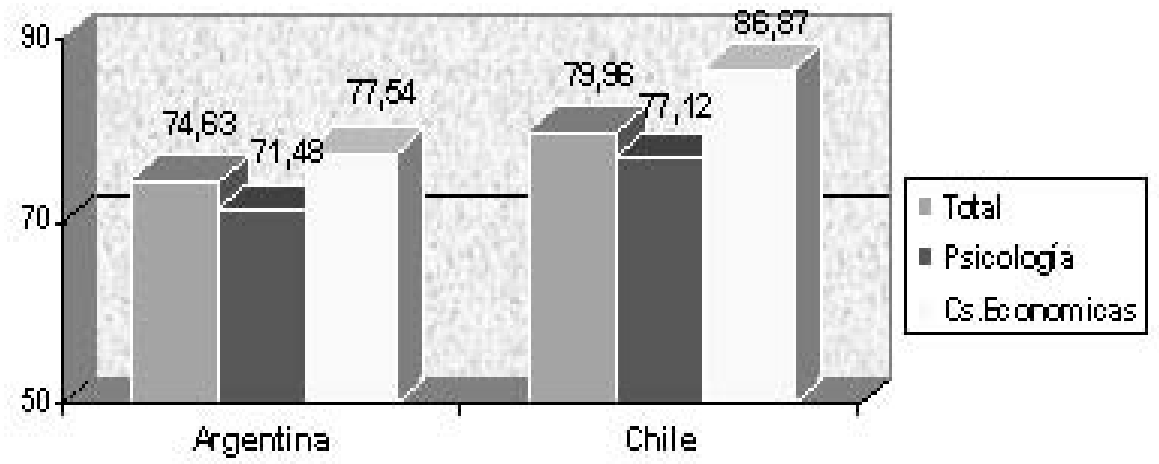

En relación a la Competitividad, como se muestra en la tabla 3, sólo se encontraron diferencias entre los grupos de estudiantes de Ciencias Económicas y empresariales de ambos países. Las puntuaciones por debajo o igual a 11 indican baja competitividad mientras que valores de 13 o más indican mayor compartir o mayor creencia en la actitud competitiva. Todas las puntuaciones alcanzan el valor de 11 o lo superan, sin embargo sólo los estudiantes de ciencias económicas y empresariales chilenos superan ampliamente la puntuación de 13 o más. Estos resultados son similares en forma parcial a los reportados por Hofstede $(1998)$, en donde Chile $(11,6)$ aparece más competitivo que Argentina $(8,6)$, aunque a la vez se observa respecto de aquellos datos un aumento en el perfil de competitividad de ambos países.

Tabla 3: Puntuaciones Medias en Competitividad por Países y Submuestras de Carreras

\begin{tabular}{c|cccc}
\hline País & & Media & D.T & $t$ \\
\hline Argentina & & 11,72 & 3.98 & \\
Chile & & 12,45 & 3.93 & n.s \\
Carrera de Estudio & País & Media & D.T & $t$ \\
Psicología & Argentina & 10,97 & 3.89 & $\mathrm{~ns}$ \\
Ciencias Económicas & Chile & 11,51 & 3.93 & \\
& & & & -2.60 \\
& Argentina & 12,41 & 3.95 & $\mathrm{P}<.01$ \\
\hline
\end{tabular}

Como puede verse en el gráfico 6, los estudiantes de ciencias económicas y empresariales chilenos aparecen más competitivos que sus pares argentinos. Este dato complementa lo mencionado previamente acerca de la Autodirección, está presente en ambos grupos pero es mayor en los chilenos, y lo mismo sucede con la Competitividad, que aparece como importante 
para ambos grupos pero en mayor medida en los estudiantes chilenos. Vale recordar que la actitud competitiva se asocia a una mayor motivación de logro, un mayor interés por el dinero y una mayor preocupación por el ahorro (Mendoza, 2004).

Gráfico 6: Puntuaciones medias de estudiantes de Ciencias Económicas de Chile y Argentina en Competitividad.

\section{(1= Fuerte Desacuerdo/ 5= Fuerte Acuerdo)}

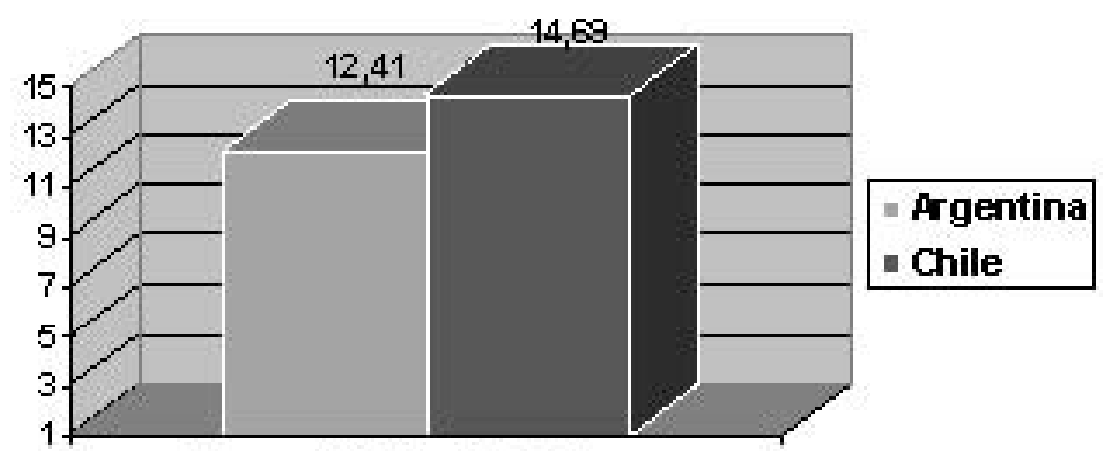

Competitividad- Cs. E con.

3.3. Relación entre Valores, EPT y Competitividad

Tabla 4: Correlaciones de Pearson entre EPT, Competitividad

y Subdimensiones de Valores

\begin{tabular}{l|cc}
\hline & COMPETITIVIDAD & EPT \\
\hline COMPETITIVIDAD &, $258(* *)$ \\
AUTOTRASCENDENCIA &,$- 119\left(^{*}\right)$ &,- 022 \\
AUTOPROMOCIÓN &, $534(* *)$ &, $191(* *)$ \\
CONSERVACIÓN &, 095 &, $148(*)$ \\
APERTURA AL CAMBIO &, $159(* *)$ &, $146(*)$ \\
\hline \multicolumn{2}{r|}{$*$ La correlación es significativa al nivel 0,01 (bilateral). } \\
\multicolumn{2}{c}{$*$ La correlación es significante al nivel 0,05 (bilateral). }
\end{tabular}

Para indagar en las relaciones que subyacen a las diferencias encontradas en los grupos analizados se realizaron correlaciones de Pearson entre las variables dependientes. Como observamos en la tabla 4, la Competitividad y la EPT mantienen una asociación positiva, ratificando la asociación reportada por Mendoza (2004) entre la EPT y la escala de Competitividad de Spence y Helmreich.

La Competitividad a su vez mantiene una fuerte asociación positiva con la Autopromoción y un 
poco más leve con la Apertura al Cambio. La asociación es negativa con la Autotrascendencia. Respecto de la EPT, también la asociación es negativa con aquella última subdimensión aunque no es significativa. La asociación es positiva con la Apertura al cambio y la Autopromoción pero también con la Conservación.

Estos resultados son similares a los encontrados por Zubieta (2007), dando fuerza a la asociación de la Competitividad con la Autopromoción, con fuerte énfasis en el individuo. Asimismo, la EPT aparece como una creencia que, centrada en el individuo -Apertura al Cambio y Autopromociónmantiene una relación a su vez con la Conservación.

\section{Discusión}

Comparando las muestras de estudiantes por países, encontramos que el grupo chileno muestra primordialmente valores que apuntan a la Apertura al cambio, es decir un mayor énfasis por la independencia de juicio y acción y, la importancia de la novedad y los desafíos. Estos valores están en oposición con los valores de Conservación, en los que incluye a la Tradición, donde todavía parece que estos sujetos mantienen un fuerte apoyo, en términos de metas motivacionales en competencia, el mayor peso en la autonomía y la mayor centralidad de lo individual parece estar en tensión con la aún importante necesidad de priorizar el mantenimiento de ciertas costumbres o normas culturales.

Algo similar ocurre con el grupo argentino; en estos sujetos el valor Seguridad adquiere una importante relevancia, valor que apunta a la conservación pero también con una meta motivacional en competencia o que sería un opuesto, el Hedonismo, todo aquello que tenga que ver con la gratificación sensual para uno mismo y el placer.

Ambos grupos muestran valores de mayor énfasis individual compitiendo con otro que prioriza las necesidades comunitarias, el grupo chileno orientándose al respeto hacia las costumbres culturales y, el grupo argentino más dirigido hacia necesidades básicas que requieren tanto los individuos como los grupos, la armonía y estabilidad, tanto de la sociedad como de las relaciones y del sí mismo.

Se relacionaba estas configuraciones, en el caso chileno, con su movimiento hacia un Individualismo Cultural que pone en discusión cierta relación sujeto-grupo y, en el caso argentino, al contexto social producido poco antes de la aplicación del cuestionario. La fuerte crisis económica, política, social vuelve a los individuos hacia una mayor Conservación, expresada en el valor Seguridad, aun con presencia de metas motivacionales más idiosincrásicas como es el hedonismo. Recordemos que de Latinoamérica, Argentina es uno de los países más individualistas, aun con el movimiento que aleja a Chile de un fuerte colectivismo (Zubieta et al., 1998). Asimismo, en la escala Val, área laboral, este grupo sobresalía en el ítem indicador de Conformismo que, en el esquema teórico de Schwartz (1992) integra, junto a la Seguridad, la dimensión subyacente de Conservación.

En lo que hace a la Ética Protestante del Trabajo y la Competitividad, el grupo de estudiantes chilenos muestra puntuaciones más altas que el grupo argentino. Como indica Mendoza (2004), el acuerdo con la EPT se asocia a la Distancia Jerárquica y el Colectivismo de Hofstede (2001) siendo una de las explicaciones más probables la referida al carácter más conservador de estas culturas. El conservadurismo se asocia en general con la EPT, dados los recursos escasos y la legitimidad de las diferencias de recompensas en estas sociedades, las personas tienden a valorar más el trabajo y el esfuerzo - particularmente los miembros de la elite de esas sociedades y los estudiantes universitarios-. Con la competitividad sucede algo similar, los estudiantes de países menos desarrollados, más colectivistas y jerárquicos, que forman parte de una elite, tienden a otorgar más importancia al dinero y al ahorro y, al mismo tiempo, muestran más actitudes más competitivas (Mendoza, 2004; p. 330).

De forma coherente con los datos de Hofstede (1998), Chile aparece como más Competitivo que Argentina. En relación a la EPT, si bien este autor no tiene datos de estos países, podemos decir que el grupo de Argentina la comparte en menor medida que el grupo de Chile. Lo mismo podría decirse de las submuestras, aunque en la puntuación los estudiantes de Ciencias Económicas de Chile son quienes mayor acuerdo muestran con esta creencia. 
Es al introducir la carrera de estudio, al igual que en el estudio previo (Zubieta, 2007) donde aquellos valores se reordenan de manera interesante. En lo que hace a los estudiantes de psicología, el grupo chileno prioriza más la Tradición mientras que el argentino prioriza el Hedonismo. Aquí, los futuros psicólogos chilenos aparecen más pendientes de lo valorado culturalmente moviéndose en el continuo individuo-grupo más a favor de este último. Por su parte, los futuros psicólogos argentinos toman el extremo inverso del continuo favoreciendo el extremo del individuo. Los estudiantes chilenos tienen una puntuación más alta en EPT pero no hay fuertes diferencias en Competitividad.

Respecto de los estudiantes de ciencias económicas, el grupo chileno muestra mayor énfasis en la Apertura al Cambio (Autodirección y Estimulación) pero también en el Poder, apareciendo la dominancia sobre personas y recursos como una meta motivacional con fuerza. También es este grupo chileno quien mayor puntuación muestra respecto de la EPT y la Competitividad. Sin embargo, son los grupos de estudiantes de esta carrera, en ambos países, los que mayores puntuaciones en EPT y Competitividad obtienen, superando a sus respectivas muestras totales de país.

Esto se podría relacionar con lo que la teoría de la Dominancia Social expresa respecto a la socialización institucional. Según la teoría, hay instituciones reforzantes de las jerarquías y la dominancia y otras, por el contrario, atenuantes. Un estudio realizado en Francia encontró diferencias entre estudiantes de abogacía y psicología en Francia (Sidanius, Pratto, van Laar y Levin, 2004).

Lo hasta aquí descrito cobra fuerza al revisar las relaciones subyacentes entre las variables de creencias y valores analizadas. Al igual que en el estudio previo con estudiantes argentinos (Zubieta, 2007), la EPT aparece asociada positivamente a la Competividad, la Autopromoción y la Conservación y, relación que antes no se encontraba, a cierta Apertura al Cambio, pero no a la Autotrascendencia. Es un conjunto de creencias que hace hincapié en el Individuo, y no en metas que incorporen fuertemente el bienestar de los demás. La competitividad, por su parte, está fuertemente asociada de forma positiva a la Autopromoción, a la Apertura al Cambio y negativamente a la Autotrascendencia, reforzando lo dicho previamente en la EPT y su orientación al individuo. A diferencia de la EPT, no se asocia positivamente a la Conservación aunque en estos datos no es significativa. Dando fuerza aún parcial a lo dicho en el trabajo previo (Zubieta, 2007), tanto la EPT como la Competitividad no se orientan al bienestar de los demás y hacen punta en el individuo pero mientras la EPT integra metas de Conservación, la Competitividad no. Y esto se desagrega cuando se analizan las submuestras de estudiantes.

Sin dejar de remarcar el hecho de que las muestras analizadas son no probabilísticas intencionales y de dos ciudades de características diferentes, como es el caso de Antofagasta, del norte de Chile, y de Buenos Aires, ciudad capital de Argentina, y que estos datos preliminares no deben ser generalizados ni ser tomados como extractos exactos de la cultura de ambos países, consideramos al estudio realizado como una buena forma de impulsar investigaciones empíricas que iluminen aspectos psicosociales relevantes para comprender la idiosincrasia de las distintas culturas y subculturas de la región latinoamericana. Asimismo, desde la perspectiva del trabajo y su dimensión psicosocial, profundizar en los valores característicos de distintas formaciones universitarias puede constituir información de gran interés para su anclaje profesional y el desarrollo de carrera de las organizaciones. En ambas líneas seguiremos trabajando. 


\section{Referencias}

Ahmed, S y Rojas Méndez, S. (1998). Estudio Comparativo de los valores en el trabajo de los estudiantes de negocios chilenos y franceses-canadienses. Revista UNIVERSUM, 13, 7-20.

Baguma, P y Furnham, A. (1993). The protestamt work ethic in Great Britain and Uganda. Journal of Cross-Cultural Psychology, Vol. 24, ํㅜ 4, 495-507.

Beit-hallami, B. (1979). Personal and social components of the Protestant Ethic. Journal of Social Psychology, 109, 263-267.

Carballo, M. (2005). Valores culturales al cambio del Milenio. Buenos Aires: Nueva Mayoría.

Casullo, M.M y Castro Solano, A. (2004). Valores Humanos y Contextos en Población Civil y Militar, Revista Acción Psicológica. Vol. 3, No 1, pp. 21-30, Ed. Servicio de Psicología Aplicada, Facultad de Psicología, UNED, Madrid, España, Octubre.

Filippi, G y Zubieta, E. (2003) Valores y Trabajo: un estudio con estudiantes universitarios. Anuario de Investigaciones 2003. Instituto de Investigaciones, Facultad de Psicología, Universidad de Buenos Aires. ISSN: 0329-5885. 2004.

Furnham, A. (1982). The protestant work ethic and attitudes towards unemployment. Journal of Occupational Psychology, 55, 277-286.

Furnham, A. (1987). Predicting Protestant work ethic believes. European Journal of Personality, 1, 93-100.

Furnham, A. (1990). The Protestant Work Ethic. London: Routledge.

Furnham, A. y Heaven, P. (1999). Personality and social behavior. Londres/Nueva York: Arnold/ Oxford University Press.

Hofstede, G. (1999). Niveles de Cultura. En G. Hofstede, Cultura y organizaciones. El Software mental. Madrid: Alianza.

Inglehart, R. (1998). Modernización y posmodernización. El cambio cultural, económico y político en 43 sociedades. Madrid: CIS/Siglo XXI.

Lynn, R. (1991). The secret of the miracle economy: Different national attitudes to competitiveness and money. Londres: Sage.

MacDonald, A. (1971). Correlates of the ethic personal conscience and the ethics of social responsibility. Journal of Consulting and Clinical Psychology, 37, 443.

McClelland, D.C. (1968). La sociedad ambiciosa. Madrid: Guadarrama.

Meda, D. (1998). El trabajo. Un valor en peligro de extinción. Barcelona: Gedisa.

Mendoza, R. (2004). Cultura y Actitudes vinculadas a la Ética Protestante, a la Competición y a la Creencia en el Mundo Justo. En D. Páez; I. Fernández; S. Ubillos y E. Zubieta. Psicología Social, Cultura y Educación. Madrid: Pearson-Prentice Hall.

Mirels, H. y Garret, J. (1971). Protestant ethic scale as a personality variable. Journal of Consulting and Clinical Psychology, 36, 40-44.

Páez, D. y González, J.L. (2000). Culture and social psychology. Psicothema, Vol. 12, Supl., 6-15.

Páez, D; Zubieta, E. (2004). Cultura y Psicología Social. En D. Páez; I, Fernández; S. Ubillos, E. Zubieta (coordinadores). Psicología Social, Cultura y Educación. Madrid: Pearson- Prentice Hall.

Pastor Ramos, G. (1986). Los grandes factorialistas de la ideología. En G. Pastor Ramos, Ideologías. Su medición psicosocial. Barcelona: Editorial Herder. 
Peiró, J. M.; Prieto, F. y Roe, R. (1996). El Trabajo como fenómeno psicosocial. En J.M. Peiró y F. Prieto. Tratado de Psicología del Trabajo, Volumen II. Madrid: Síntesis.

Prieto, F.; Peiró, J.M.; Bravo, M.J. y Caballer, A. (1996). Socialización y Desarrollo del Rol Laboral. En J.M. Peiró y F. Prieto. Tratado de Psicología del Trabajo, Volumen II. Madrid: Síntesis.

Rokeach, M. (1973). The nature of human values. Nueva York: Free Press.

Schein, E. (1972). Professional Education: Some New Directions New York: McGraw-Hill.

Schein, E. (1978). Career Dynamics: Matching Individual and Organizational Needs. Reading, MA: Addison Wesley Publishing Company.

Schein, E. (1990). Career Anchors. San Diego, CA: University Associates.

Schein, E. (1996) Psicología de la Organización. México: Ed. Prentince Hall.

Schwartz, S. (1992). Universal in the content and structure of values: Theoretical advances and empirical test in 20 countries. En P. Zanna (comp.). Advances in Experimental Social Psychology, vol.25, Nueva York: Academic Press.

Schwartz, S. (1992). Universals in the content and structure of values: Theoretical advances and empirical tests in 20 countries. En M. P. Zanna (Ed.), Advances in experimental Social Psychology (Vol. 25, pp. 1-65). Nueva York: Academic Press.

Schwartz, S. (2001). ¿Existen aspectos universales en la estructura de los valores humanos? En Ros, M; Gouveia, V. Psicología Social de los Valores Humanos. Desarrollos teóricos, metodológicos y aplicados. Madrid: Biblioteca Nueva.

Schwartz, S. (2005). Basic Human Values: Their content and structure across countries. En A. Tamayo y J. Porto (eds.), Valores y trabalho [Values and work]. Brasilia: Editora Universidade de Brasilia.

Sidanius, J; Pratto, F; van Laar, C. y Levin, S. (2004). Social Dominance Theory: Its agenda and Method. Political Psychology, Vol. 25, Nº, 845-880.

Triandis, H. C. (1994). Culture and Social Psychology. Nueva York: McGraw Hill.

Weber, M. (1985). La ética protestante y el espíritu del capitalismo. México: Premia Editora.

Zubieta, E. (2007). Creencias en la Ética Protestante del Trabajo (EPT) y Competición en estudiantes universitarios. Aceptado para su publicación en Perspectivas en Psicología, Vol. IV, Noviembre de 2007.

Zubieta, E.; Fernández, I.; Vergara, A.; Páez, D.; Martínez, M. y Candia, C. (1998). Cultura y Emoción en América, Boletín de Psicología Social, $N^{\circ} 61$, Valencia, España. 